\title{
Chemical Bonds between Charged Atoms in the Even-Odd Rule and a Limitation to Eight Covalent Bonds per Atom in Centered-Cubic and Single Face-Centered-Cubic Crystals
}

\author{
Geoffroy Auvert1 ${ }^{1}$, Marine Auvert ${ }^{2}$ \\ ${ }^{1}$ Grenoble Alps University, Grenoble, France \\ ${ }^{2}$ University of Strasbourg, Strasbourg, France \\ Email: Geoffroy.auvert@grenoble-inp.org
}

Received 27 August 2015; accepted 1 November 2015; published 4 November 2015

Copyright (C) 2015 by authors and Scientific Research Publishing Inc.

This work is licensed under the Creative Commons Attribution International License (CC BY). http://creativecommons.org/licenses/by/4.0/

\section{(c) (i) Open Access}

\section{Abstract}

A crystal is a highly organized arrangement of atoms in a solid, wherein a unit cell is periodically repeated to form the crystal pattern. A unit cell is composed of atoms that are connected to some of their first neighbors by chemical bonds. A recent rule, entitled the even-odd rule, introduced a new way to calculate the number of covalent bonds around an atom. It states that around an uncharged atom, the number of bonds and the number of electrons have the same parity. In the case of a charged atom on the contrary, both numbers have different parity. The aim of the present paper is to challenge the even-odd rule on chemical bonds in well-known crystal structures. According to the rule, atoms are supposed to be bonded exclusively through single-covalent bonds. A distinctive criterion, only applicable to crystals, states that atoms cannot build more than 8 chemical bonds, as opposed to the classical model, where each atom in a crystal is connected to every first neighbor without limitation. Electrical charges can be assigned to specific atoms in order to compensate for extra or missing bonds. More specifically the article considers di-atomic body-centeredcubic, tetra-atomic and dodeca-atomic single-face-centered-cubic crystals. In body-centered crystals, atoms are interconnected by 8 covalent bonds. In face-centered crystal, the unit cell contains 4 or 12 atoms. For di-element crystals, the total number of bonds for both elements is found to be identical. The neutrality of the unit cell is obtained with an opposite charge on the nearest or second-nearest neighbor. To conclude, the even-odd rule is applicable to a wide number of compounds in known cubic structures and the number of chemical bonds per atom is not related to the valence of the elements in the periodic table. 


\section{Keywords}

\section{Even-Odd, Rule, Covalent Bond, Single Bond, Crystal, Solid, Centered, Face-Centered, Unit Cell}

\section{Introduction}

In crystal structures, positions of atoms in the unit cell of a crystal are derived from experimental diffraction data [1]. Given the atoms positions, the coordination number of each atom, i.e. the number of its near neighbors can be evaluated [2]. For molecules, this number also indicates the number of bonds around the atom [3] [4]. Various types of bond can bind an atom and its first neighbors [5]. Unfortunately, diffraction data do not give direct information to choose the nature of bonds in a solid [6]. For the same reason, there is no direct information about atoms charges.

The recently proposed even-odd rule gives a novel procedure to evaluate and draw bonding configurations and precisely locate charges in ions [7] and molecules [8]. This procedure has been firstly successfully tested on well-known single bonded ions and molecules and secondly shown capable of turning multi-bonded ions and molecules into single-bonded compounds [9]. This procedure indicates on which atoms charges are located in charged or uncharged compounds. Applied to ions and molecules, it also gives the number of covalent bonds that each atom may form in agreement with its valence number [10].

The objective of the present paper is two-fold: to validate first an extension, of the procedure to cubic crystalline structures, during which we show that the number of bonds is not limited to the valence number; then to propose alternative bonding configurations in well-known cubic structures while limiting the number of covalent bonds per atom to a maximum of eight.

Two types of atom configurations in cubic crystalline structures are described without bonds: centered-cubic and single face-centered cubic. The even-odd rule is defined and a constraint in the number of covalent bonds for each atom is introduced: no atom should form more than eight covalent bonds. The rule and constraints are then applied to both crystalline structures taking example of a large number of known compounds. Results are discussed and the authors conclude on the validity of the even-odd rule for crystals including the maximum of 8 bonds for each element.

In the present paper, compounds and structures names are found in "crystallography open data base" [11], atoms positions in unit cells in "Wikipedia" [12]. Unit cells, atoms and bonds are represented thanks to "Avogadro V1", 3D molecular structure editor [13], completed with a drawing software to indicate charges [14].

To keep this publication consistent with the previous three [7]-[9], the writing of chemical formulas is for example: CaF2 for calcium fluoride and $\mathrm{HfO} 2$ for hafnium oxide and in a crystal, both are described as di-elements tri-atomics unit cells.

\section{Centered and Face-Centered Crystals}

Crystal structures are represented as a pattern of periodically repeated unit-cells. A unit cell is the base structure, classically represented as contained in a small imaginary box [15]. This cell can have different configurations [15] [16]. The present article focuses on cubic unit cells as shown in Figure 1, in which the cube is symbolized by red edges showing 8 corners, 6 faces and 12 edges.

Atoms can be arranged in many different ways inside cubic unit cells. In order to keep this article clear and concise, the authors resolved to focus on two of them. The first one is a centered-cubic structure, Figure 1(a), and the second is a single-face-centered-cubic structure, Figure 1(b). In Figure 1(a) and Figure 1(b), each colored sphere represents an atom. Atoms can lie in the center of the cube, in the center of faces or in corners.

In Figure 1(a), the central atom in the cube has a neighbor in a corner of the cube. When the unit cell is repeated, the same central atom ends up having 8 near neighbors, one in each corner of the cube. In the same way, an atom in the corner of the cube has 8 near neighbors, located in the center of each of the 8 cells it belongs to.

In Figure 1(b), four atoms can be seen: one is in a corner and the other three are face centered. The corner atom seems to have three first-neighbors in the same cell but only a half of each belongs to that cell. When the unit cell is repeated, the total number of first-neighbors is 12 . Atoms located in the center of a face have 12 near neighbors as well. 


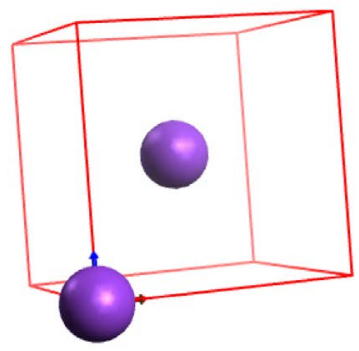

(a)

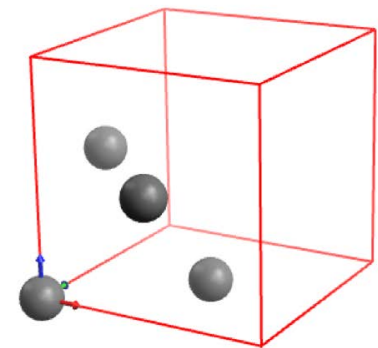

(b)

\begin{abstract}
Figure 1. Cubic unit cells in crystals showing two specific arrangements of atoms. In (a) a centered-cubic unit cell with two atoms per cell and in (b), a simple-face-centered-cubic unit cell with four atoms per cell. (For a representation with all atoms participating in the unit cell, refer respectively to Figure 2 and Figure 4).
\end{abstract}

Although X-ray diffraction can indicate the arrangement of atoms in a crystal, covalent bonds and potential charges cannot be derived with any known method. In the following, a rule is described to systematize how to draw bonds between atoms in cubic unit cells. This procedure uses a line to represent a single-covalent interconnection between two first-neighbor-atoms.

\title{
3. The Even-Odd Rule in a Procedure to Draw a Valid Unit Cell with Single-Covalent-Bonds between Charged or Neutral Atoms
}

The even-odd rule was developed for molecules and ions under liquid and gaseous phase [7]-[9]. In this chapter the even-odd rule is described when applied to solid crystals.

In the present article and in the context of the even-odd rule, the authors define the following expressions: 1) even-atom means an atom with an even number of electrons and 2) odd-atom means an atom with an odd number of electrons. For instance, reading the number of electrons in the periodic table, carbon is an even-atom and nitrogen is an odd-atom.

In scientific literature treating of crystalline structures, atom positions and their bonding arrangement in the unit cell are available for some cubic crystals [12]. Other crystal descriptions, however, totally lack information on charge or interconnection locations [17]. A six-step, clear and well-defined procedure based on the even-odd rule is proposed below, aiming to systematize how to check validity of bonding configuration in crystals:

\section{- Interconnections between atoms}

- Every multiple-bonds must be replaced by a single-covalent bond as described in ref. [9].

- When an atom has more than eight covalent bonds, remove connections in excess down to eight.

- When an atom is charged, the charge affects the number of possible bonds: As an example: $\mathrm{C}$ has 4 or 2 bonds then $\mathrm{C}(+)$ must be with 3 or 1 bonds [7].

\section{- Even-odd rule}

- Uncharged even-atoms must have an even number of bonds

- Uncharged odd-atom must have an odd number of bonds

- If an even-atom or an odd-atom is charged, the reverse condition must be used

- In order to meet the above conditions, one can modify the charge or the number of bonds.

- Unit cell on the whole neutrally charged

- Charged atoms must be balanced in the unit cell by opposite charges on a first or second neighbor.

- The total charge of the crystal must be zero; when possible, this is applied to each unit cell; if not, the cell charge is neutralized by one of the nearest unit cell.

\section{- Conservation of the total number of electrons}

- All bonds must be covalent, i.e. involve one pair of electrons.

- The total number of electrons in the cell, once bonds are drawn, must be identical to the total number of electrons of each atom without bond as given by the periodic table.

\section{- Charge and bonding conditions}

- Atoms bearing identical charges cannot be connected

- When a unit cell contains more than one element, two atoms of the same element are not interconnected. 
- The consequence on studied structures is:

o In mono-element crystals with di- or tetra-atomics unit cells, each atom counts the same number of bonds and neighbors are neutral or bear opposite charges.

0 In di-elements crystals with di-atomics unit cells, each atom counts the same number of bonds and two neighbors are neutral or with opposite charges in first or second neighbor.

0 In di-elements crystals where unit cells count more than 4 atoms, the total number of bonds of the first element is equal to the total number of bonds of the second element. The neutrality of the unit cell is obtained inside one series of elements or between both series.

- When disconnecting two atoms does not provide a satisfactory solution, extend the unit cell to an even number of the base unit cell previously used.

- The number of bonds of an element in a crystal does not need to match with its valence number of the periodic table.

- Geometry of unit cells in a crystal:

- Opposing faces of a unit cell are identical.

\section{The Even-Odd Rule Applied to Centered-Cubic Crystals}

The even-odd rule indicates how to draw bonds in a crystal and to adapt the charge of every atom to its bonding configuration. In this chapter, the rule is applied to centered-cubic crystals with 8 covalent bonds for each atom in the unit cell.

\subsection{Centered-Cubic Crystals with Mono-Element Di-Atomic Unit Cell}

The objective is to build a covalent bonding configuration in a centered-cubic structure (see Figure 1(a) for the bondless structure). Figure 2(a) depicts a center-cubic structure with atoms located in the center of the unit cell and in its corners. The central atom belongs to the visible unit cell and each atom at the corner belongs to 8 unit cells, in other words only $1 / 8$ of a corner atom belongs to the visible unit cell. Figure 2(a) also shows singlecovalent bonds between each nearest neighbor. With 8 nearest neighbors, the central atom has eight single-covalent bonds and a corner-atom has also eight covalent bonds, one in each unit cell it belongs to. According to the even-odd rule, since atoms in the current structure have an even number of bonds:

- If the crystal is composed of even-atoms, (Figure 2(a)), atoms composing the structure are uncharged. As listed in the right-hand column of Figure 2(a), main group elements falling into this category are Barium (56), Calcium (20), Radium (88), Selenium (34) and Strontium (38). Chromium, Iron and Molybdenum are few examples in the category of transition elements.

- If the crystal is composed of odd-atoms, (Figure 2(b)), they must be charged, either positively or negatively. With a charge, the number of electrons for each atom becomes evidently even and atoms can build an even number of covalent bonds. In order to neutralize the charge borne by the central atom on the scale of a unit cell, atoms located in corners of the cube must bear an opposite charge. As detailed in the right-hand column of Figure 2(b), from the main group of the periodic table, six elements can form this type of structure: Antimony, Cesium, Potassium, Sodium, Rubidium and Thallium. In the group of transition elements: Europium, Manganese, Niobium, Neptunium, Vanadium and Tantalum can take this configuration.

All 26 elements listed in Figure 2(a) and Figure 2(b), have an Im-3m structure [11]. They are drawn with the procedure described previously, i.e. their bonding and charge organizations are in agreement with the even-odd rule.

\subsection{Centered Cubic-Crystals with Di-Element Di-Atomic Unit Cell}

Figure 3 depicts three centered-cubic structures with a di-element di-atomic cell. The structure name is $\mathbf{P m}-\mathbf{3 m}$ [11]. For each case, compounds from ref. [11] that can adopt this structure are listed in the right-hand column.

In Figure 3(a), the binary compound is composed of neutral even-atoms and each atom has eight bonds, the unit cell is consequently uncharged. In Figure 3(b), with a compound composed of odd-atoms, one atom is positively charged and the other is negatively charged; the unit cell is consequently also neutrally charged. In Figure 3(c), binary compounds are composed of one odd-atom and one even-atom. Only odd-atoms bear a charge, since they are evenly bonded. The neighboring even atoms are also evenly bonded, so they cannot bear a charge. Compensating the charge borne by the odd-atoms is thus only possible at a greater scale, i.e. if the odd-atom of 


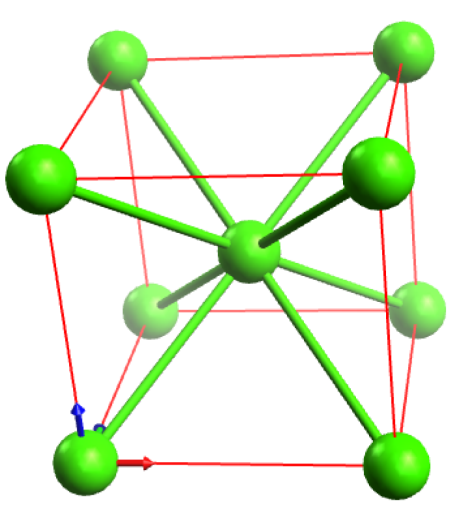

(a)

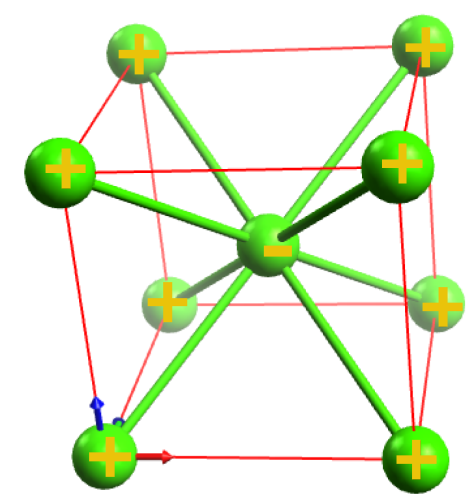

Uncharged even-atoms

Structure Im-3m [11]

Elements from the main group.

Ca Beta 20 c2 [11] [13]

Ba 56 [11]-[13]

$\mathrm{Ra} 88$ c2 [11] [12]

Se 34 [11]

Sr 38 [11]

Transition metals

Cr 24 [11]-[13]

Fe 26 [11]-[13]

Mo 42 [11]-[13]

Pu 94 [11] [13]

Ti 22 [11] [13]

Th 90 [11]

U 92 [11]

Zr 40 [11]

W 74 [11] [12]

\author{
Charged odd-atoms in neutral unit-cell \\ Structure Im-3m [11] \\ Elements from the main group \\ Cs 55 [11]-[13] \\ K 19 [11]-[13] \\ $\mathrm{Na} 11$ c1 [11]-[13] \\ $\mathrm{Rb} 37$ [11] [12] \\ Sb 51 c5 [11] \\ Tl alpha 81 c3 [11] [12]
}

\title{
Transition metals
}

Eu 63 [11]-[13]

Mn 25 [11]-[13]

$\mathrm{Nb} 41$ [11]-[13]

Np 93 [11]-[13]

V 23 [11]-[13]

Ta 73 [11] [12]

(b)

Figure 2. Unit cell of a centered-cubic structure with two atoms per cell in an $\mathbf{I m}-\mathbf{3 m}$ structure. In (a), all even-atoms are neutral and count 8 covalent bonds, in (b), all oddatoms count 8 covalent bonds but bear alternatively opposite charges allowing for a neutral unit cell.

the nearest unit cell bears an opposite charge. The opposite charge is on the second nearest neighbor placed in the nearest unit cell.

In this chapter, all 75 binary compounds, found in ref. [11], adopt a Pm-3m structure. They have been processed with the procedure described previously and they agree with the even-odd rule. Elements studied belong to all groups of elements in the periodic table and all count 8 bonds. It seems to confirm the theoretical hypothesis according to which the number of bonds of an element in a cubic crystal is independent from the valence number of the said element.

The next structure studied is a single-face-centered cubic-structure: $\mathbf{F m}-\mathbf{3 m}$ or $\mathbf{P m}-\mathbf{3 m}$.

\section{Single-Face-Centered Cubic-Crystals with a Limited Number of Bonds}

The even-odd rule will be applied first to be consistent with the chemical formula of the compound and second to use, when needed, a maximum of 8 covalent bonds for all atoms in the unit cell.

\subsection{Single-Face-Centered Cubic-Crystals with Uncharged Mono-Element Tetra-Atomic Unit Cell}

A single-face-centered cubic crystal contains 4 atoms per unit cell. (This word "single” is used to discriminate this structure from a double-face-centered crystal that involves 8 atoms in a unit cell). This single-face-centered 


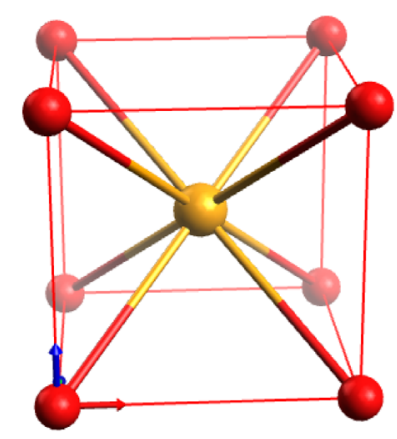

(a)

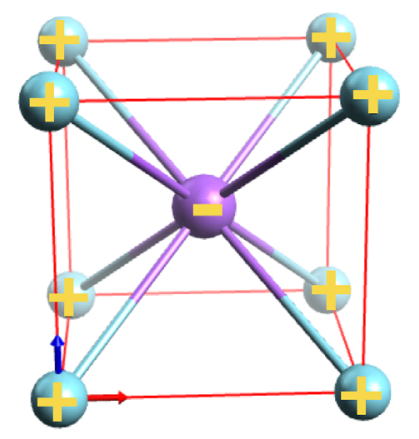

\begin{tabular}{lll}
\multicolumn{3}{l}{ Odd-atoms/odd-atoms } \\
\multicolumn{2}{l}{ Structure } & \\
AuCs & $-3 m[11]$ & \\
AuHo & $\mathrm{AgLa}$ & $\mathrm{ClK}$ \\
$\mathrm{AuLu}$ & $\mathrm{AgPr}$ & $\mathrm{ClRb}$ \\
$\mathrm{AuPr}$ & $\mathrm{AgSc}$ & $\mathrm{ClTl}$ \\
$\mathrm{AuRb}$ & $\mathrm{AgTb}$ & $\mathrm{CsI}$ \\
$\mathrm{AuSc}$ & $\mathrm{AgTm}$ & $\mathrm{ITl}$ \\
$\mathrm{AuTb}$ & $\mathrm{BrCs}$ & $\mathrm{NI}$ \\
$\mathrm{AuTm}$ & $\mathrm{BrN}$ & $\mathrm{NaCl}$ \\
$\mathrm{AuY}$ & $\mathrm{BrTl}$ & $\mathrm{SbTl}$ \\
$\mathrm{AgGa}$ & $\mathrm{ClN}$ & \\
$\mathrm{AgHo}$ & $\mathrm{ClCs}$ & \\
$\mathrm{AgI}$ & &
\end{tabular}

(b)

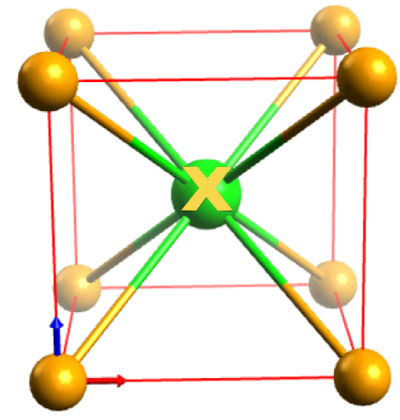

\begin{tabular}{|c|c|c|c|}
\hline \multicolumn{4}{|c|}{$\begin{array}{l}\text { Even-atoms/odd-atoms or odd-atoms/even-atoms } \\
\text { Structure } \mathrm{Pm}-3 \mathrm{~m}\end{array}$} \\
\hline $\mathrm{AgCd}$ & $\mathrm{AuGd}$ & $\mathrm{AuZn}$ & $\mathrm{CsS}$ \\
\hline $\mathrm{AgCe}$ & $\mathrm{AuMg}$ & $\mathrm{BrTi}$ & $\mathrm{CuPd}$ \\
\hline AgDy & AuNd & CsS & $\mathrm{CuZn}$ \\
\hline $\mathrm{AgEr}$ & $\mathrm{AuSm}$ & $\mathrm{CaTl}$ & $\mathrm{HgLi}$ \\
\hline $\mathrm{AgGd}$ & $\mathrm{AuTi}$ & $\mathrm{CdLa}$ & $\mathrm{LaMg}$ \\
\hline $\mathrm{AgMg}$ & $\mathrm{AuYb}$ & $\mathrm{CdPr}$ & $\mathrm{LaZn}$ \\
\hline $\mathrm{AgNd}$ & $\mathrm{AlNi}$ & $\mathrm{CoFe}$ & $\mathrm{MgPr}$ \\
\hline $\mathrm{AgYb}$ & $\mathrm{AuCd}$ & $\mathrm{CsSe}$ & MgTl \\
\hline $\mathrm{AgZn}$ & AuDy & & $\mathrm{NbO}$ \\
\hline AlNd & $\mathrm{AuEr}$ & & $\operatorname{PrZn}$ \\
\hline
\end{tabular}

(c)

Figure 3. Centered-cubic crystals with a di-element di-atomic unit cell. In (a), both atoms are even-atoms and none bear charges. In (b), both atoms are odd-atoms and bear an opposite charge, i.e. + and - for a neutral unit cell. In (c), an atom in the corner of the unit cell is an uncharged even-atom and the center odd-atom bears a charge. The total charge of the cell is neutralized by the second neighbor odd-atom in the nearest unit cell.

cubic crystal is depicted in Figure 4 with a complete set of identical atoms extended from the unit cell of Figure 1(b).

In Figure 4(a), atoms locations are displayed in the mono-element tetra-atomic unit cell, without showing covalent bonds. Each atom counts 12 neighbors. In the present article the number of covalent bonds is however proposed to be limited to a maximum of 8 covalent bonds. Figure 4(b) shows the bonding configuration in agreement with the even-odd rule and the limitation rule: there are no horizontal connections and each evenatom is uncharged.

Mono-element compounds listed in the right-hand column of Figure 4(b) have the same structure [11]. It includes noble gases as Argon, Krypton, Neon and Xenon, known in chemistry for not binding easily. In this chapter they build 8 covalent bonds. This shows again that, in cubic crystals studied in this article, the valence 


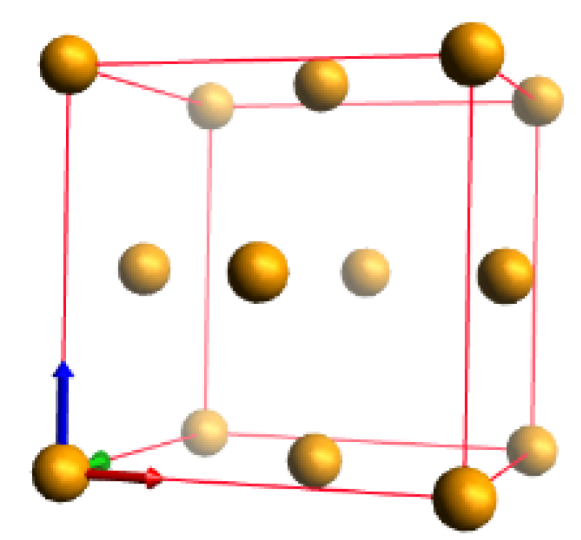

Single-face-centered cubic crystals

Structure: Fm-3m [11]

Mono-element tetra-atomic unit cell

$3 \mathrm{D}$ view of a cubic unit cell without bonds.

Only 4 atoms belong to this unit cell.

14 atoms involved in one unit cell.

Six atoms are face-centered and eight are corners atoms.

Each atoms counts 12 first neighbors.

(a)

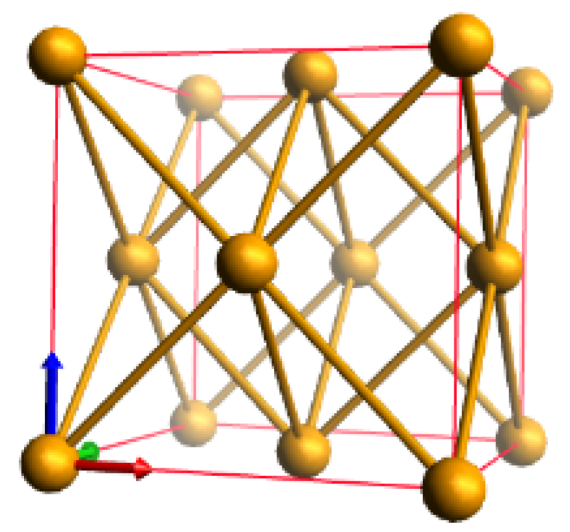

Bonding procedure

All atoms count 8 single-covalent bonds, regardless of their location

in the unit cell.

Even-atoms from the main group

$\operatorname{Ar} 18$ [11]-[13]

Ba 56 [12]

Ca alpha 20 [11]-[13]

$\mathrm{Kr} 36$ [11]-[13]

Ne 10 [11]-[13]

Sr 38 [11]

Rn 86 [11]

Xe 54 [11]-[13]

Transition-Metals even-atoms

Fe gamma 26 [11]-[13]

Ni 28 [11] [12]

$\mathrm{Pb} 82 \mathrm{c} 4[11]$

Pt 78 [11]-[13]

Pd 46 [11]-[13]

Yb Beta70 [11]-[13]

(b)

Figure 4. Face-centered cubic structure in a tetra-atomic unit cell. In (a), 14 atoms are shown without any bond and in (b), each even-atom is interconnected to eight of its 12 neighbors in a nearly vertical direction and without charge.

number has no impact on the number of bonds between atoms.

\subsection{Single-Face-Centered Cubic-Crystals with Charged Mono-Element Tetra-Atomic Unit Cell}

The same single-face-centered cubic-crystal structure can be composed of odd-atoms. In order for the odd-atoms to build an even number of covalent bonds, the even-odd rule states that atoms must be charged. Figure 5 depicts the interconnection system allowing this $\mathbf{F m}-\mathbf{3 m}$ structure to be in agreement with the even-odd rule. Atoms belonging to a horizontal plane are not connected and bear the same charge. Each successive plane bears alternatively positive and negative charges in order to obtain neutral unit cells. Figure 5 also shows that each covalent bond connects atoms with opposite charge, i.e. no two atoms bearing the same charge are bonded. The number of covalent bonds for each atom is also compliant and equal to 8. It is also noteworthy that, for all mono-element di-atomic compounds in the right-hand side column of Figure 5, the number of bonds is not related to the valence number of the elements involved.

\subsection{Single-Face-Centered Cubic-Crystals with Di-Element Tetra-Atomic Unit Cell}

Following structures with a different structure name: $\mathbf{P m}-\mathbf{3 m}$ and with the same single-face-centered cubic organization, comes from available data [11]. Four different configurations of di-elements tetra-atomics unit cells are detailed in Figure 6 and Figure 7. 


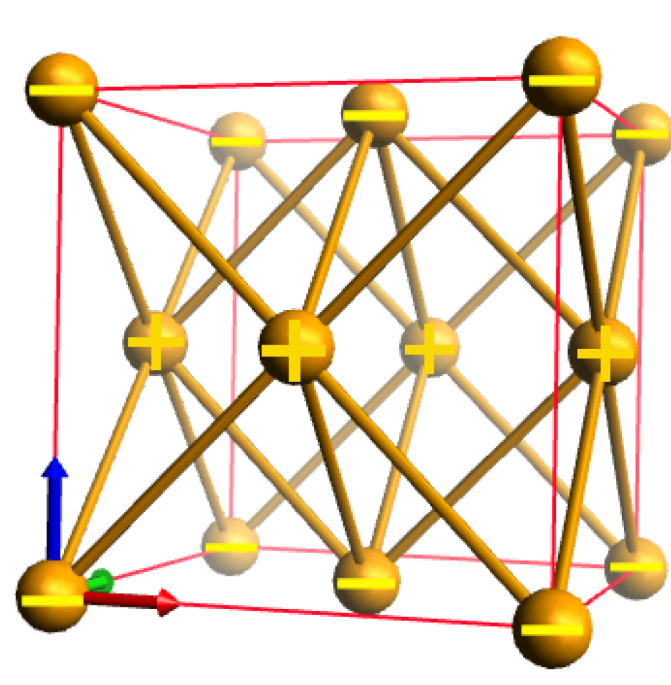

Odd-atoms in single-face-centered cubic crystals

Structure: Fm-3m

Each atom counts 8 covalent bonds

Elements from the main group

Al Alpha 13 [11]-[13]

Na 11 [12]

Transition-Metals

Ac 89 [11]-[13]

Ag 47 [11]-[13]

Am Alpha 95 [11]

Au 79 [11]-[13]

$\mathrm{Cu} 29$ [11]-[13]

Ir 77 [11]-[13]

Mn gamma 25 [11] [13]

Rh 45 [11]-[13]

Connections only between + and - charges.

Neutral unit cell.

Figure 5. Face-centered cubic-crystals with a mono-element tetra-atomic unit cell. 14 atoms are shown, each with 8 covalent bonds. The odd-atoms are charged to allow an even number of bonds. Notice that atoms with the same charge are not interconnected.

FeNi3: Fe brown, Ni Green

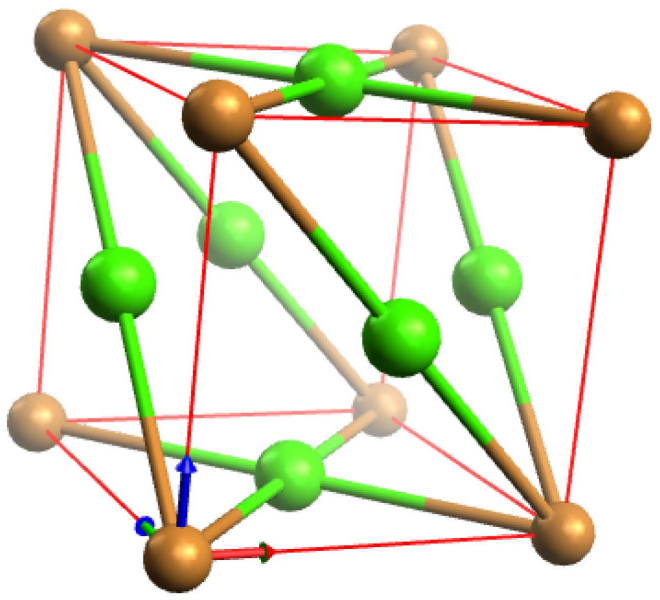

Even-even atoms

Structure: Pm-3m

Fe: 8 bonds in corners

$\mathrm{Ni}$ face centered

Bonds: $2 * 2+4=8$

Neutral atoms

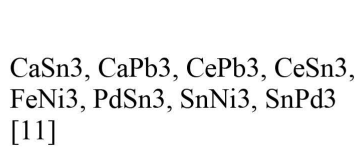

[11]
NaPb3: Na brown, Pb Green

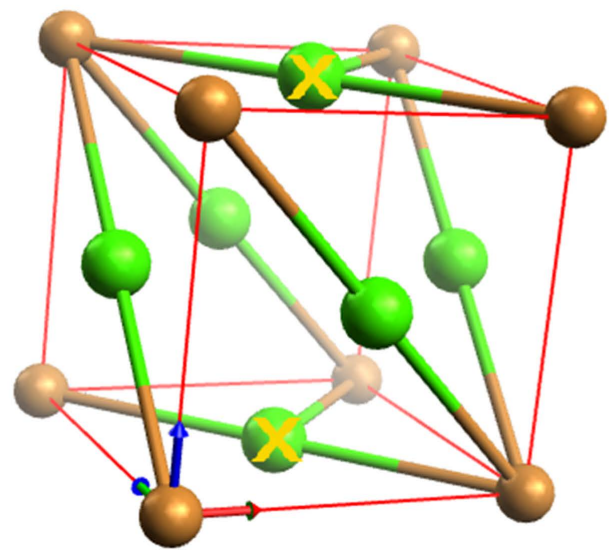

Odd-even atoms

Structure: Pm-3m

$\mathrm{Na} 7$ bonds and neutral

$\mathrm{Pb}$ face centered

Bonds: $1 * 3+2 * 2=7$

One charged $\mathrm{Pb}$ atom

(a)

AuTi3, InPt3, LaSn3, NaPb3, $\mathrm{ReO} 3$ [11]

(b)

Figure 6. Single-face-centered crystals with di-element tetra-atomic unit cell. In (a), Fe atoms (brown) have an even number of electrons and they are uncharged with an even number of bonds. Ni atoms (green) are uncharged even-atoms. In (b), Na atoms (brown) are odd-atoms with an odd number of bonds (7 for one atom). $\mathrm{Pb}$ atoms (green) are neutral even-atoms with two bonds and charged with three bonds and the same total number of bonds (7 for three green atoms).

In Figure 6(a), FeNi3 is an example of the first case: the di-element tetra-atomic unit cell is composed of even-atoms. Inside, the iron atom (brown) counts 8 bonds. Two nitrogen atoms (green) have two bonds and one four bonds with a total of 8, which corresponds to the number of bonds of the iron atom. Eight compounds, listed below the drawing, have this configuration.

In Figure 6(b), NaPb3 is an example used as a reference of an odd-even configuration. Na atoms (brown) are 
PtCu3: Pt brown, Cu Green

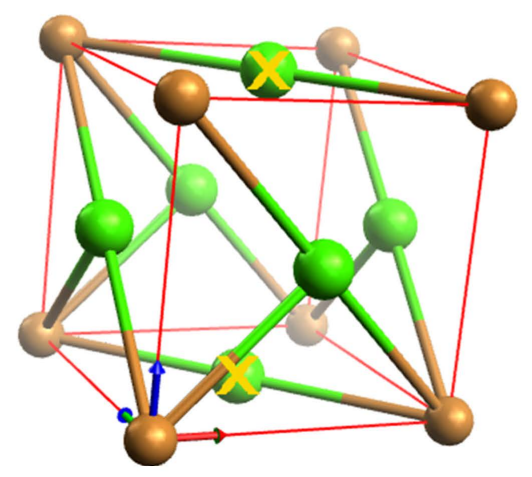

Even-atom/odd-atom Structure: Pm-3m Orange Pt: $16 / 2=8$ bonds Green $\mathrm{Cu}$ : Bonds: $1 * 2+2 * 3=8$
One charge per unit cell. Neutralized in nearest unit cell.

CaTI3, CeIn3, ErGa3, MgAg3, PtAg3, PtCu3 [11]

(a)

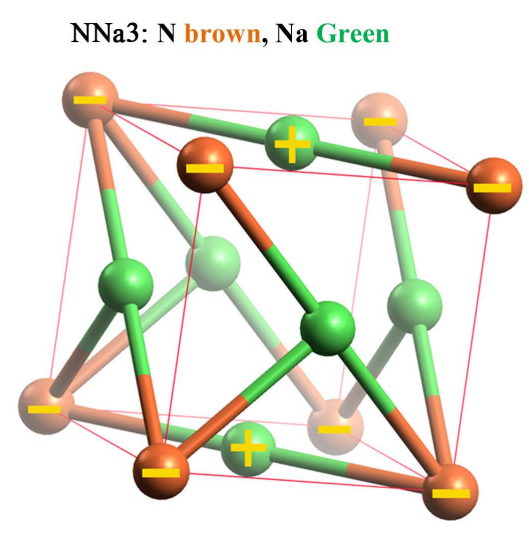

Odd-atoms/odd-atoms. Structure: Pm-3m $\mathrm{N}$ in corners $16 / 2=8$ bonds $\mathrm{Na}$ Face-centered atoms Bonds: $1 * 2+2 * 3=8$
Neutral charge in planar layer $\mathrm{AuCu} 3, \mathrm{CuAu} 3, \mathrm{GaMn} 3, \mathrm{InAg} 3$, LiAu3, NCu3, NNa3, TmAg3, TmGa3, YF3 [11]

(b)

Figure 7. Single-face-centered crystals with 4 atoms per unit cell. In (a), one even-atom (brown) and 3 odd-atoms (green) per unit cell. In (b), one odd-atom (brown) and 3 odd-atoms (green) per unit cell. The charge positions and number of bonds follow the even-odd rule.

odd-atoms and are uncharged thanks to an odd number of bonds. With 7 bonds, it is neutral in Figure 6(b). Inside the unit cell, all three $\mathrm{Pb}$ atoms (green) should have the same total number of bonds. This is possible with 2 bonds for two $\mathrm{Pb}$ atoms and 3 for the third one as depicted in Figure 6(b). The Pb with 3 bonds, being an evenatom, is charged and it cannot be neutralized in the cell. This charge, located in the center of the horizontal face, can only be compensated in the nearest unit cell by nearest second neighbor in the same horizontal plane. Five compounds, listed below the drawing, have this configuration.

After these even-even and odd-even configurations, the even-odd and odd-odd configurations are shown in Figure 7. In Figure 7(a), PtCu3 is an example used as a reference of an even-odd configuration. Pt atoms (brown), located in corners of the cell, are even-atoms, have 8 bonds and are uncharged. Of the Cu odd-atoms (green), one is charged and bonded twice whereas both others are uncharged and bonded three times. Note that inside the unit cell, the total count of Cu-atoms bonds is 8, which corresponds to the number of bonds of the Pt atom. The atom located in the center of the lower horizontal place is charged and is compensated in the nearest unit cell. Below the PtCu3 crystal, a list of 6 compounds that can have this bonding configuration [11].

In Figure 7(b), NNa3 is shown as a reference for two odd-atoms. Nitrogen atoms (brown), located in a corner has 8 bonds and must be charged. Of the three Nickel odd-atoms (green), two are uncharged with 3 bonds and one is charged with two bonds. It gives the eight expected bonds inside the unit cell. The neutrality of each horizontal planes ensures neutrality of the crystal. Ten binary compounds take this configuration and their formulas are referenced [11].

The limitation to a maximum of eight bonds per atom is very important in these four configurations. It imposes a particularly strong constraint on the second atoms present three times in the compound. In a di-elements tetra-atomics unit cell represented by AB3, the corner atoms A count 8 or 7 bonds and are interconnected with the three other elements with a total number of bonds inside the unit cell equal to 8 or 7 respectively. Each B atom can thus not build many bonds.

In the following, the total number of atoms per cell is extended to 12 atoms without any change in the single-face-centered cubic structure: Fm-3m.

\subsection{Single-Face-Centered Cubic-Crystals with a Dodeca-Atomic Unit Cell}

The present structure has the same face-centered atom positions with a tetra-atomic unit cell but a series of 8 atoms is added in the unit cell. They form a di-element dodeca-atomic unit cell with an Fm-3m structure as shown in Figure 8 and Figure 9. 


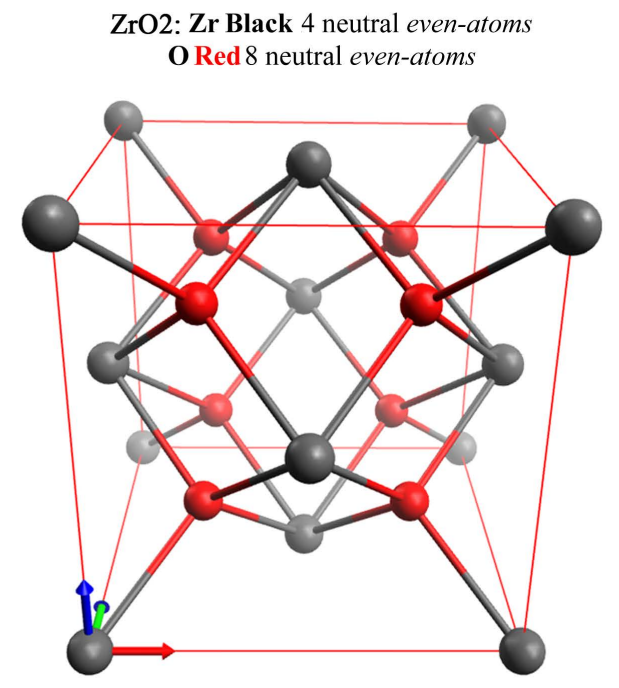

Binary compounds in Fm-3m structure: [11]

$\mathrm{CeO} 2, \mathrm{CmO} 2, \mathrm{GeMg} 2, \mathrm{HfO} 2, \mathrm{Mg} 2 \mathrm{~S}, \mathrm{Mg} 2 \mathrm{Si}$, NiSi2, $\mathrm{PbO}$, $\mathrm{PbMg} 2 \mathrm{PoO} 2, \mathrm{PtSn} 2, \mathrm{PuO} 2, \mathrm{SnMg} 2, \mathrm{ThO} 2, \mathrm{UO} 2, \mathrm{ZrO} 2$

(a)

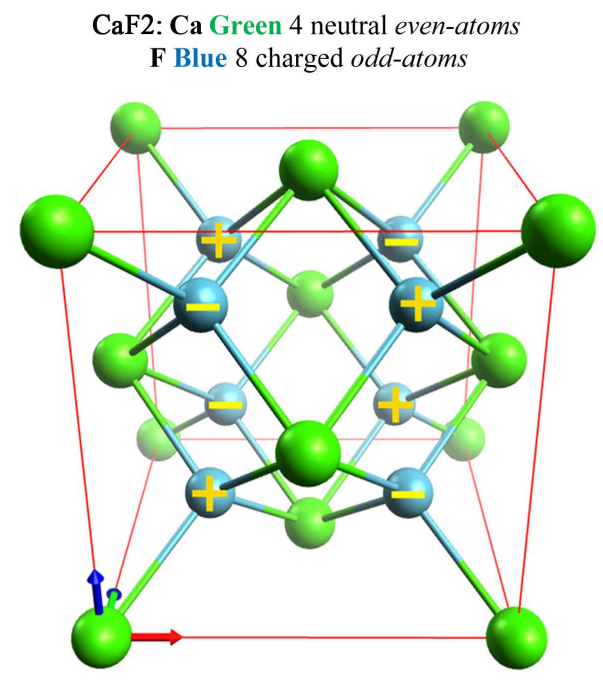

Binary compounds in Fm-3m structure: [11]

$\mathrm{BaCl} 2, \mathrm{BaF} 2, \mathrm{CaF} 2, \mathrm{CdF} 2, \mathrm{HgF} 2, \mathrm{OK} 2, \mathrm{OLi} 2, \mathrm{ONa} 2, \mathrm{SLi} 2, \mathrm{SNa} 2$, $\mathrm{PbF} 2$, PtAl2, PtGa2, PtIn2, PtK2, RaF2, SK2, SRb2, SeK2, SeCu2, SeLi2, SeNa2, SrF2, SrCl2, TeAg2, TeK2, TeLi2, TeNa2, UN2

(b)

Figure 8. Face centered unit cell with 8 added atoms in Fm-3m structure, giving a dodeca-atomic unit cell. In (a), interconnections between neutral even-atoms. In (b), all eight internal atoms are charged, with $4(+)$ and $4(-)$, and the total charge is neutralized.

PrO2: Pr Grey, 4 odd-atoms with a neutral total charge Oxygen Blue 8 neutral even-atoms

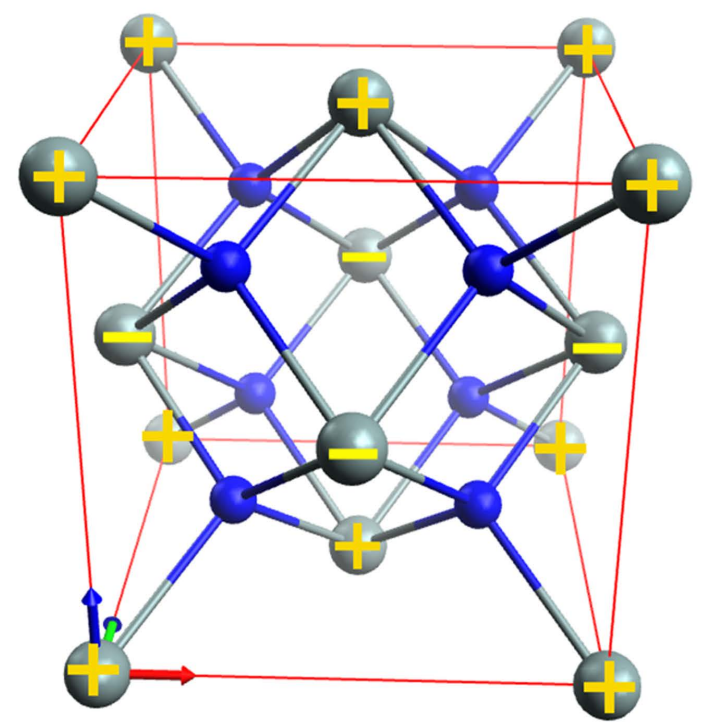

Binary compounds, structure Fm-3m: [11] $\mathrm{CoSi} 2, \mathrm{EuC} 2, \mathrm{IrSn} 2, \mathrm{NpO} 2, \mathrm{PrO} 2, \mathrm{TbO} 2$

(a)
EuF2: Eu Green Odd-atoms with a neutral total charge Fluorine Blue Odd-atoms: neutral with 3 bonds

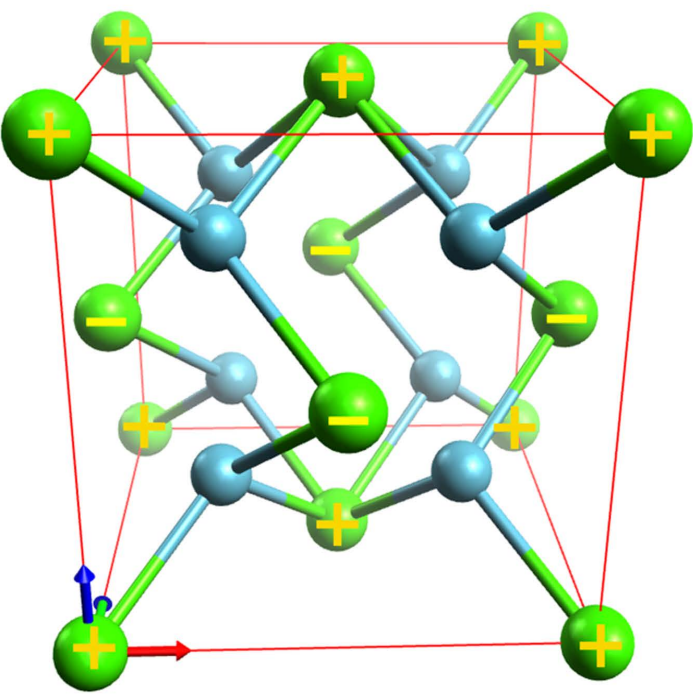

Binary compounds, structure Fm-3m: [11]

AuGa2, AuAl2, AuIn2, AuSb2, EuF2, NpN2, PIr2, PRh2.

(b)

Figure 9. Face-centered unit cell with face-centered cubic structure: Fm-3m and 8 atoms added to the unit cell with a total number of 12 atoms. (a) shows neutral internal atoms and atoms charges in the face-centered structure, (b) has nearly the same structure than (a), except with uncharged odd-atoms and a reduced number of covalent-bonds. 
In Figure 8(a), a well-known chemical formula with this face-centered structure is $\mathrm{ZrO}$. $\mathrm{Zr}$ even-atoms (black) are face-centered and oxygen even-atoms (red) are added inside the unit cell. Red atoms are only connected to black atoms and vice versa. Red atoms have 8 times 4 bonds and black atoms balance them with 8 times 1 bond and 6 times 4 bonds. In Figure 8(a), oxygen even-atoms count 4 bonds each. As listed at the bottom of Figure 8(a), 16 compounds follow this structure and most of them are dioxides.

The eight uncharged oxygen even-atoms of Figure 8(a) can be replaced by charged odd-atoms. The unit cell neutrality is only reached thanks to the even number of internal atoms. Figure 8(b) illustrates such a structure, of which $\mathrm{CaF} 2$ is an example. Ca atoms are green and $\mathrm{F}$ atoms are blue. The bonding condition is unchanged compared to Figure 8(a), i.e. the structure is conserved. It can also be described like a face-centered crystal with eight internal halogen atoms. As calcium is an even-atom and drawn with eight single covalent bonds, it is consequently neutral and follows the even-odd rule. On the contrary, fluorine is an odd-atom and must be charged to allow four covalent bonds. Of all fluorine atoms, four are positively charged and four negatively. This is confirmed in Figure 8(b) where charges are assigned to build a neutral unit cell. There are about 30 three-atomic compounds conforming to this structure [11]. Their names appear at the bottom of Figure 8(b).

The same configuration can be obtained when replacing the $\mathrm{Zr}$ even-atom of Figure 8(a) with an odd-atom. Figure 9(a) illustrates the result, an odd-even configuration. To conserve a neutral total charge in the unit cell of Figure 9(a), horizontal planes of odd-atoms are alternatively positively and negatively charged (as in Figure 5). Six di-element dodeca-atomic unit cells from ref: [11] follow the proposed bonding structure.

The last possible change from Figure 8(a) is to replace all even-atoms by odd-atoms. A difficulty arises when attempting to keep the same bonding configuration since it would result in interconnected atoms bearing the same charge. To avoid this, each blue odd-atom must lose one covalent bond. As a consequence, green atoms must lose two bonds to keep the same charge and the same bonding parity, as shown in Figure 9(b). An example is EuF2, in which all eight Fluorine odd-atoms (blue), have 3 bonds and therefore no charge. The total number of bonds from the fluorine is 24 ( 8 times 3). The number of bonds with Eu odd-atoms is 24 ( 8 times 1 for the corners, 2 times 4 for the horizontal faces and 4 times 2 for vertical faces centered atoms). This neutral structure is consistent with the even-odd rule. Eight di-element compounds listed below Figure 9(b) have this Fm-3m structure [11].

In Chapter 5, 45 compounds were found in scientific literature to adopt this single-face-centered-cubic structure based on a dodeca-atomic unit cell. They all are compatible with the even-odd rule and the limitation to 8 bonds per atom. With another point of view in this crystals, an element presents four times has 8 or 6 bonds interconnected directly to an element presents 8 times with 4 or 3 bonds respectively. According to the proposed procedure, the neutrality of the unit cell is obtained, when necessary via the nearest second neighbor placed in the same unit cell.

\section{Discussion}

More than 180 referenced configurations of atoms in body-centered cubic and simple-face-centered cubic structures have been studied in this article. This number seems great enough to form a solid base for the following discussion.

Crystals are highly organized structures in which a unit-cell is repeated over and over. Atoms are likely to form this type of structure only if there is only one possibility for them to connect. If there were more than one possible configuration, crystals would not be as regular as they are naturally found. The proposed procedure was thus built to constrain validity of bonds enough to leave only one possible bonding configuration. The limitations are: single covalent bonds only; atoms erect a number of bonds as allowed by the even-odd rule, depending on their charge and their even or odd character; no element can form more than 8 bonds; two atoms bearing the same charge, positive or negative, cannot be connected; in mono-element crystals, two atoms are connected when they are both neutral or with complementary charges; a unit cell with series of elements have connections only between different atoms; a unit cell with two series of elements have the same total number of bonds in each series; a unit cell containing charged atoms should be overall neutrally charged, or should be neutralized by the nearest neighbor or by the second nearest neighbor in the nearest unit cell.

Some of these points are now discussed below.

\section{- Validity of the procedure based on the even-odd rule}

The drawing procedure described above was systematically tested on single-covalent bonded structures in 
centered-cubic crystals and single-face-centered cubic crystals. The list of compounds and their structures were found in ref. [11] with the following criteria: 1) di-atomic unit cell with mono-element and di-elements cubic structure (Pm-3m and Im-3m); 2) tetra-atomic unit cell with mono-element and di-elements (Fm-3m and Pm-3m), 3) dodeca-atomic unit cell with di-elements cubic structure (Fm-3m). For all listed compounds, a bonding configuration was found by applying the procedure. It seems safe to conclude that, after having been previously applied successfully to molecules [8] and ions [7], the even-odd rule is applicable to the named cubic structures.

\section{- Limitation to a maximum of 8 covalent bonds per atom in crystals}

The limitation to a maximum of 8 covalent bonds seems to be commonly accepted in chemistry. In solid chemistry however, this limitation is neither applied nor considered. In many crystals, atoms are surrounded by more than 8 atoms and connections are usually drawn between all nearest neighbors. In this article however, the limitation to 8 bonds per atom has gradually appeared necessary as the authors applied the drawing procedure to all typical even-odd configurations in di-atomic compounds: even-even, even-odd, odd-even and odd-odd. By limiting the number of bonds to a maximum of 8 , the number of possible bonding configurations decreased, but one possible configuration always remained.

\section{- Particularity of alkaline earth metals.}

Elements of column 2 in the periodic table can be sorted into two groups, Ca, Sr and Ba form face-centered crystals whereas Be and $\mathrm{Mg}$ do not. Beryllium possesses only four electrons in total, which explain why it cannot build 8 covalent bonds as needed in centered cubic crystals. Magnesium on the other end possesses 12 electrons and no explanation for this inability to build 8 covalent bonds was satisfying at the time of writing.

\section{- Particularity of halogen in mono-element body-centered-crystals.}

When non-metals halogen odd-atoms ( $\mathrm{F}, \mathrm{Cl}, \mathrm{Br}$ and I) crystallize, they never seem to adopt body-centered structures. The reason for this peculiar case could be that they cannot build more than seven bonds, as in IF7 molecules.

\section{- Inner shell electrons}

In chemistry, electrons located in the inner shell do not participate in the formation of bonds. A neutral atom cannot form more bonds than the number of electrons in its valence shell. Sodium has, for instance, one electron in the valence shell—it is an odd-atom — and ten in the inner shell. Neutrally charged, a Sodium atom only erects one bond as in $\mathrm{NaOH}$. In a crystal structure like the mono-element cubic structure, however, Sodium atoms are bonded 8 times. Since the single valence electron can only participate in one covalent bond, the only possible explanation for the other bonds is a contribution of electrons from the inner shell. Because only pairs of electrons can be extracted from the inner shell, the Sodium atoms alternatively use 6 or 8 electrons from the inner shell and are charged to build an even number of bonds.

In crystals, the valence number clearly does not limit the number of bonds. The present paper proposes to extend to other elements the need of an even number of electrons coming from the inner shell to form bonds in cubic crystals.

\section{- Cleavage of crystals}

A crystal is cleaved along oriented planes [18]. Silicon crystals, diamond-like, have three main cleavage directions. Silicon crystals, mono-element crystals made out of even-atoms with four bonds, do not contain charged atoms. In other crystals containing charged atoms, charges are aligned on planes and cleavage probably occurs perpendicularly to these electrostatic planes. It would be of great interest to confirm that cleavage directions in crystals studied in the present article are the same for crystals with the same bonding configuration and differ according to the electrostatic planes.

\section{Conclusions}

The even-odd rule, checked previously for ions and molecules, is applied in this paper to centered-cubic and face-centered-cubic crystals. The bonding organization for mono-atomic and diatomic crystals is drawn using the even-odd rule in body-centered crystals, giving evidence of the quality of the rule. In the same way, di-element compounds in single-face-centered crystals are clearly described and, when needed, charges are assigned to atoms to build a structure of 8 covalent bonds or less.

The proposed constraint for a maximum of 8 covalent bonds per atom, is plausible for such structures, because it brings similarity to other areas of chemistry on the one hand and because it reduces the number of possible bonding configurations on the other hand. With the even-odd rule and the added constraints, every solid 
compounds found in literature that fit the chosen criteria were systematically studied and a bonding configuration was found. This appears to form a large support for the acceptance of the even-odd rule.

It will be interesting to extend the drawing procedure described here for single-face-centered crystals, to the very common double-face-centered structure.

\section{References}

[1] http://en.wikipedia.org/wiki/Crystallography

[2] http://en.wikipedia.org/wiki/Coordination_number

[3] http://chemwiki.ucdavis.edu/Inorganic_Chemistry/Coordination_Chemistry/Coordination_Numbers

[4] http://en.wikipedia.org/wiki/Bonding_in_solids

[5] http://en.wikipedia.org/wiki/Chemical_bond

[6] https://en.wikipedia.org/wiki/X-ray_crystallography

[7] Auvert, G. (2014) Chemical Structural Formulas of Single-Bonded Ions Using the "Even-Odd" Rule Encompassing Lewis's Octet Rule: Application to Position of Single-Charge and Electron-Pairs in Hypo- and Hyper-Valent Ions with Main Group Elements. Open Journal of Physical Chemistry, 4, 67-72. http://dx.doi.org/10.4236/ojpc.2014.42010

[8] Auvert, G. (2014) Improvement of the Lewis-Abegg-Octet Rule Using an "Even-Odd" Rule in Chemical Structural Formulas: Application to Hypo and Hyper-Valences of Stable Uncharged Gaseous Single-Bonded Molecules with Main Group Elements. Open Journal of Physical Chemistry, 4, 60-66. http://dx.doi.org/10.4236/ojpc.2014.42009

[9] Auvert, G. (2014) The Even-Odd Rule on Single Covalent-Bonded Structural Formulas as a Modification of Classical Structural Formulas of Multiple-Bonded Ions and Molecules. Open Journal of Physical Chemistry, 4, 173-184. http://dx.doi.org/10.4236/ojpc.2014.44020

[10] Mendelejew, D. (1869) Über die Beziehungen der Eigenschaften zu den Atomgewichten der Elemente. Zeitschrift für Chemie (in German), 405-406.

[11] http://cod.ibt.lt/

[12] https://en.wikipedia.org/

[13] Hanwell, M.D., Curtis, D.E., Lonie, D.C., Vandermeersch, T., Zurek, E. and Hutchison, G.R. (2012) Avogadro: An Advanced Semantic Chemical Editor, Visualization, and Analysis Platform. Journal of Cheminformatics, 4, 17. http://dx.doi.org/10.1186/1758-2946-4-17

[14] http://www.gimp.org/downloads/

[15] https://en.wikipedia.org/wiki/Crystal_structure

[16] Haynes, W., Ed. Handbook of Chemistry and Physics. CRC 96th Edition.

[17] Hatert, F. Compléments de cristallographie www.minera.ulg.ac.be/pdf/

[18] https://en.wikipedia.org/wiki/Cleavage_(crystal) 